\title{
Dynamic mechanical behavior of filled rubber at small strains
}

\author{
C. Michael Roland \\ Chemistry Division, Code 6120, Naval Research Laboratory, \\ Washington, D.C. 20375-5000
}

(Received 13 April 1989; accepted 13 June 1989)

\section{Synopsis}

The strain and temperature dependence of the dynamic properties of rubber containing various concentrations of carbon black were characterized. The measurements, obtained at lower strain amplitudes $\left(<10^{-5}\right)$ than previous studies, indicate that flocculation of the carbon black particles, and the enhanced modulus and damping effected by it, are likely existent prior to any deformation. The disruption of the carbon black net work structure was found to be independent of the mechanical behavior of the polymer, occurring at the same macroscopic strain independently of the stress level. The implications of this in terms of the flocculation process are discussed. At reduced temperatures, filler interparticle interactions intensify, and consequently their contribution to the mechanical properties increases. This greater influence of the filler structure is observed even as the glass transition of the rubber is approached and the behavior of the rubber and carbon black phases converge.

\section{INTRODUCTION}

As a result of marked improvements in mechanical behavior together with significant economic advantages, rubber reinforced with carbon black represents the most widely used and extensively studied polymeric composite. The contributions of the filler to the mechanical behavior are strongly strain dependent. A portion of the modulus enhancement of filled rubber results from amplification of the strain on chain molecules in proximity to the inextensible carbon black particles. ${ }^{1-4}$ With appropriate consideration of the influence of rubber occlusion by the particles, this hydrodynamic effect accounts very well for the rheology of filled rubber at moderate strain levels. ${ }^{5-8}$ At higher strains debonding of the polymer chains from the carbon black, ${ }^{9,10}$ orientation of the latter phase, ${ }^{11,12}$ and microscopic crack deviation ${ }^{13}$ have been invoked to explain the various physical properties. 
The simplest mechanical behavior might be expected at very low strains; however, the published data are difficult to reconcile with accepted notions of the morphology of carbon black-rubber composites. At diminished strain amplitudes a significant contribution to the stiffness arises from interaction between aggregates of the carbon black. This flocculation (also referred to as agglomeration) gives rise to a filler network that pervades the rubbery continuum, and is responsible for high electrical conductivity, ${ }^{14-17}$ yield stresses (thixotropy), ${ }^{18-20}$ and a modulus inversely dependent on strain. ${ }^{2-23}$ The latter phenomena reflect the break up of the flocculated structure, as the mechanical stress overcomes the van der Waals forces responsible for bonding of the particles. Although electrical conduction can be effected by electron tunneling and hopping (thermally activated transport between traps in the polymeric medium ), ${ }^{14,16,24,25}$ and thus does not require direct contacts between the carbon black particles, nevertheless deformation brings about a loss of conductivity that parallels the loss of mechanical stiffness. ${ }^{11,26-28}$

The model of a filled elastomer as comprised of a matrix of rubber containing a network of carbon black is consistent with high electrical conductivity in the absence of strain, and with the reduction of both electrical conductivity and modulus upon deformations of sufficient magnitude to break up the flocculated structure. The modulus, of course, cannot be measured without straining. Below the level at which the filler network is disrupted, the elastic modulus is known to exhibit an invariance to deformation that is maintained down to roughly $10^{-3}$ strain amplitude. ${ }^{23,29,30}$ However, it has been reported that at lower strains filled rubber becomes more compliant, with broad maxima observed for the dependence of the dynamic modulus on strain amplitude. ${ }^{11,31-34}$ Such a decrease in modulus at very low strains is consistent with the idea of a three-dimensional network structure produced by the interaction of the filler particles only if the flocculation is brought about by deformation (presumably as the strain approaches about $10^{-3}$ in amplitude). While high unstrained electrical conductivity could still be rationalized in terms of conduction by an electron hopping mechanism without actual particle contacts, the occurrence of a yield stress in filled rubber implies that the filler network exists prior to any deformation.

The details of the strain dependence of dynamic properties has important implications regarding the morphology of carbon black reinforced rubber. The reported observations of maxima in the elastic modulus ${ }^{11.31-34}$ have been criticized as resulting from measurements made at strain amplitudes beyond the range of the experimental apparatus. ${ }^{23}$ It has been suggested that only when data have been obtained on more sensitive 
instrumentation can the apparent discrepancies between morphological models and the observed behavior of filled rubber be resolved. ${ }^{29}$ The present experiments were directed toward gaining a clearer understanding of the relationship between structure and rheology in this important class of polymeric composites.

\section{EXPERIMENTAL}

A rubber formulation was used which consisted of cis-1,4-polyisoprene (SMR-L grade natural rubber) with $1.0 \%$ and $3.0 \%$ by weight, respectively, of antioxidant (Agerite D from the R. T. Vanderbilt Co.) and dicumyl peroxide. The polymer was mixed in a Brabender Prepcenter with $18.7 \%$ to $31.5 \%$ by volume ( 45 to 90 parts per hundred rubber) of N110 carbon black (Vulcan 9 from Cabot Corp.). This is an SAF type black, with high structure and a very small particle size. Mcasurements were also made on a blend containing $25 \%$ of the polyisoprene and $75 \%$ poly (vinylethylene) with $0.5 \%$ peroxide and $27.2 \%$ by volume ( $75 \mathrm{phr}$ ) of the N110 black. The materials were cured in all cases at $160{ }^{\circ} \mathrm{C}$ for 30 min.

Mechanical testing was carried out at $30^{\circ} \mathrm{C}$ except where otherwise noted, using an Imass $\mathrm{Co}$. Dynastat Mark II Instrument. The resolution of the displacement transducer, $0.05 \mu$, enables imposition of very small strains. For the polyisoprene based samples, a tension-compression deformation cycle with zero mean load was utilized. The height $(19 \mathrm{~mm})$ and diameter $(12.5 \mathrm{~mm})$ of the bonded cylindrical test specimens provided for readily measurable loads at very small strains. The shearing at the ends which accompanies the compression of a bonded cylinder ${ }^{35}$ was negligible with the present configuration. For the blend samples testing extended to temperatures approaching the transition to the glassy state; accordingly, these experiments were made in uniaxial extension on thin rectangular strips.

Although the instrumental compliance was low $(0.001 \mathrm{~mm} / \mathrm{kg})$, correction for $\mathrm{it}^{36}$ significantly altered the obtained results, particularly for cylindrical test specimens. To improve the signal to noise ratio, data taken at the lowest strain amplitudes were averaged over 60 cycles. Viscous heating was minimized by employing a $0.1 \mathrm{~Hz}$ frequency for all testing. Individual measurements over the course of the 60 cycles confirmed the absence of any measurable thermal effects.

\section{RESULTS}

The elastic modulus $E^{\prime}$, measured for the polyisoprene with various levels of carbon black, is displayed in Fig. 1. The expected scaling at low 


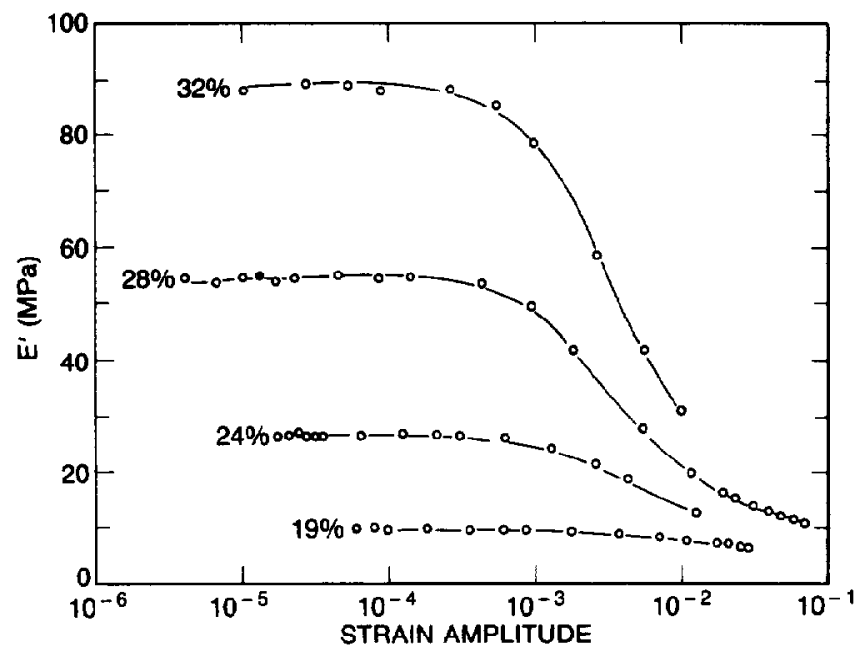

FIG. 1. The storage modulus measured (at $30^{\circ} \mathrm{C}$ and $0.1 \mathrm{IIz}$ ) for polyisoprene containing the indicated volumes of $\mathrm{N} 110$ carbon black. It is seen that $E^{\prime}$ becomes invariant at low strain.

strains with filler content is observed. At all concentrations of filler $E^{\prime}$ exhibits an invariance to strain amplitudes for strains less than about $10^{-4}$. Most significantly, the high elastic modulus known to be associated with low strain amplitudes ${ }^{23,29,30}$ is maintained down through the lowest strains at which the present measurements were made $\left(\sim 10^{-6}\right)$. This clearly suggests that the carbon black network exists at the lowest mechanical strains, consistent with observations of yield stresses ${ }^{18-20}$ and high electrical conductivity ${ }^{14-17}$ in carbon black-rubber composites. There is no hint in the present data of any strain-induced flocculation, as would be inferred from previous results suggesting maxima in the $E^{\prime}$ vs strain amplitude relation. ${ }^{11,31-34}$

The loss modulus $E^{\prime \prime}$ could be reliably measured only at strains above about $10^{-5}$. It was essentially constant prior to an increase at deformations approaching the level at which the elastic modulus markedly decreases (Fig. 2). A maximum in $E^{\prime \prime}$ in this range of strains is well established as reflecting the hysteresis arising from the breakup of the filler network. ${ }^{23,29,30}$ No indications were apparent herein of any of the multiple maxima in the loss modulus vs strain relationship reported by some investigators. ${ }^{11,31,33,34}$ 


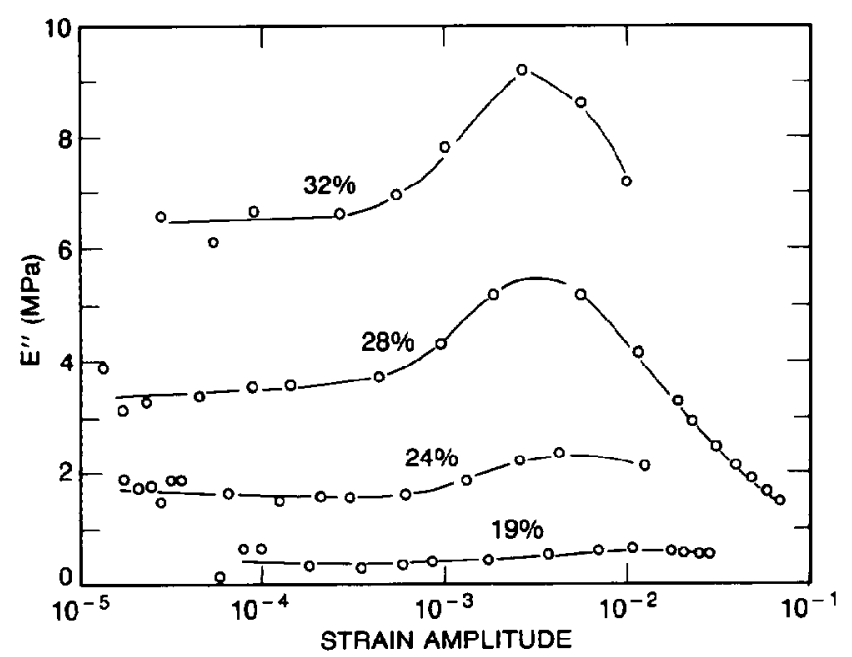

FIG. 2. The loss modulus (at $30^{\circ} \mathrm{C}$ and $0.1 \mathrm{~Hz}$ ) for polyisoprene containing the indicated volume of carbon black.

The magnitude of $E$ " at the lowest strains exceeds that at strains greater than $10^{-2}$. This indicates that a certain degree of energy dissipation is transpiring in the filler phase at strains insufficient for significant irreversible break up of the flocculated structure. Any contribution to the hysteresis from alterations in the composite morphology would not be inconsistent with an intact filler structure, provided that such processes are completely recovered during the time scale of a strain cycle. Rearrangement of the carbon black morphology without changes in the dynamic modulus have in fact been observed in combined static and oscillatory deformation experiments. ${ }^{37}$ These processes apparently leave the filler network intact, whereby $E^{\prime}$ is undiminished. Frictional drag between closely neighboring aggregates may generate hysteresis that is absent at higher deformations. It is evident that both the stiffness and the hysteresis of a filled rubber at very low mechanical strains can be expected to parallel the carbon black content and its degree of flocculation.

Nonlinear viscoelastic behavior is, of course, implicit in the results of Fig. 1; nevertheless, even at amplitudes for which the modulus is a marked function of strain (between $10^{-3}$ and $10^{-2}$ ), an undistorted sinusoidal output is obtained in response to a sinusoidal input. This proportionality 
between stress and strain under conditions whereby $E^{\prime}$ and $E^{\prime \prime}$ are strongly strain dependent is a consequence of the dynamical nature of the network disruption-formation process. ${ }^{29}$ At steady state, interaggregate bonds broken during a given deformation cycle are reformed during the same cycle; thus, at a given strain amplitude the stress and strain remain in direct proportion, albeit with a phase lag. This transience of the network structure during the course of a deformation cycle is secn directly in electrical conductivity measurements. ${ }^{11,26-28}$

To the extent that the dynamic recovery of the filler structure cannot be completed during the time scale of one deformation cycle, some dependence of the modulus-strain relationship on frequency or temperature might be expected. Williams-Landel-Ferry type transformations of temperature and frequency data have been carried out with only modest success on filled elastomers in the rubbery state at low strains, with thermal expansion coefficients and WLF shift factors found to be minimally influenced by the presence of filler ${ }^{38,39}$ The success of such superpositioning is dependent on the extent to which the temperature dependence of the relevant mechanisms (involving, for example, the segmental mobility of the polymer chains and the interactions between filler particles) are equivalent. ${ }^{29}$ The transition temperature itself is invariant to carbon black concentration. ${ }^{40,41}$ These results suggest that the segmental mobility of a polymer is minimally affected by absorption of some chains onto the carbon black surface or by thcir proximity to a filler particlc. While most characterization of the dynamic properties of carbon black-rubber composites is done at temperatures well above the glass transition temperature, it is instructive to carry out these measurements at lower temperatures.

Displayed in Fig. 3 is the strain dependence of the elastic modulus measured at a series of temperatures for a filled blend of polyisoprene and poly (vinylethylene). This blend is miscible and thus exhibits a single glass transition, ${ }^{4-44}$ that is moreover at a convenient temperature. The temperature range of the data encompasses the glass to liquid transition region of the blend (Fig. 4). The qualitative dependence of $E^{\prime}$ on strain amplitude is seen in Fig. 3 to be comparable at the various temperatures. It is particularly noteworthy that the break up of the carbon black network occurs for all temperatures at essentially the same strain. Evidently the conditions necessary for network disruption are independent of the mechanical response of the polymeric phase. The disruption of the filler structure seems to be governed only by the total strain on the composite, rather than depending, for example, on the strain energy, as has been suggested. ${ }^{45,46}$ A strain amplitude criterion for network disruption has 


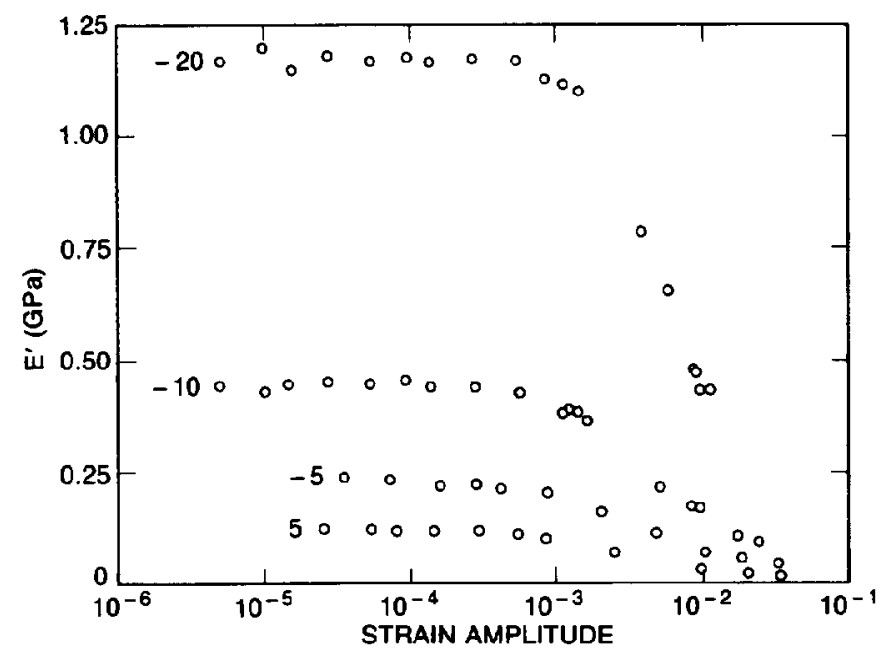

FIG. 3. The storage modulus for a blend of $75 \%$ poly (vinylethylene) and $25 \%$ polyisoprene with $27 \%$ by volume of $\mathrm{N} 110$, measured at various temperatures (denoted in Celsius alongside the respective curves). As the temperature approaches that of the glass transition of the rubber matrix, the contribution of the filler to the low strain mechanical behavior is enhanced.

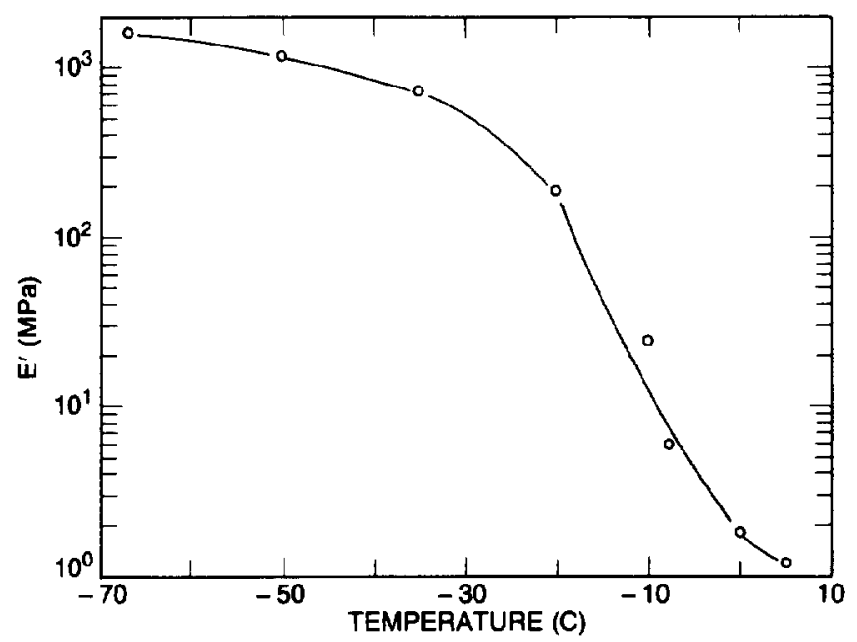

FIG. 4. The elastic modulus of an unfilled blend of $75 \%$ poly (vinylethylene) and $25 \%$ polyisoprene measured at $0.1 \mathrm{~Hz}$. 
successfully been employed to describe measurements on a wide range of carbon black-rubber composites. ${ }^{29,47}$ Taking into account such results, a model has been developed in which both the critical strain required for network disruption and the strain dependence of the breakage rate of the aggregate contacts are assumed to be universal constants. ${ }^{47}$ Implicit in such a model is the further assumption that the respective rate constants for floccuation and break up of the floc have the same (or no!) dependence on stress. However debatable such assumptions may be, the data in Fig. 3 are compatible with this model. It might be expected that the local strain on the filler phase, rather than the overall composite strain, should control aggregate debonding. If the mechanical interactions in filled rubber coincided with parallel coupling of the filler and polymeric phases, the two criteria would be equivalent. ${ }^{48}$ Direct measurement of microscopic strains in the two phases would be of interest in this regard.

It is observed in Fig. 3 that the magnitude of the modulus enhancement from the carbon black network increases markedly as the temperature is reduced toward that of the glass transition. This behavior conforms to previous observations made at temperatures strictly in the rubbery region. ${ }^{23,49}$ Under appropriate conditions the electrical conductivity of filled rubber will also decrease with increases in temperature, with the effect, however, ascribed to the change of interaggregate distances by thermal expansion. ${ }^{14,16,37,50}$ If the dissociation of the aggregate contacts is thermally activated, the increase in low strain modulus upon cooling is an expected consequence of escalating flocculation. ${ }^{47,51}$ The low strain elastic modulus vs temperature data exhibit positive deviations from Arrhenius behavior, indicating that while an equilibrium in the extent of flocculation may exist, the composite modulus is further augmented at lower temperature by the increasing stiffness of the polymer phase. The contribution from the filler nevertheless increases markedly with a reduction in temperature, giving rise to a more strain dependent modulus. This in direct contradiction to the prediction, predicated upon a closer equivalence of the respective moduli of the filler and polymer in the transition region, of a convergence there of the low and high strain moduli of carbon black reinforced rubber ${ }^{47}$ Some earlier experimental results, which suggested that filled and unfilled rubbers would become more similar as the transition region was approached, were either done at high strain amplitude $^{41}$ or were confused by failure to maintain a constant strain amplitude. ${ }^{38}$ As Fig. 3 clearly illustrates, both the effects of strain and temperature on dynamic properties are significant. The more glass-like behaviur of the polymeric phase at lower temperatures is evidently accompanied by increased filler stiffness due to greater aggregate interaction. 


\section{CONCLUSIONS}

The experimental data described herein suggest that a carbon black network structure exists in filled rubber absent any deformation. As a consequence the dynamic properties are independent of strain for strain amplitudes below about $10^{-3}$. The high modulus and increased energy dissipation associated with very low strain deformations are largely independent of the mobility of the polymer segments, notwithstanding the interaction of the latter with the carbon black. The onset of glassy behavior in the polymer does not appreciably diminish the strong strain dependence of the dynamic properties of carbon black-rubber composites. At least at very low strains, the proliferation of interaggregate contacts continues to dominate the mechanical response.

\section{ACKNOWLEDGMENTS}

Enlightening discussions with Mr. J. D. Ulmer of the Firestone Tire and Rubber Company are gratefully acknowledged. This work was supported in part by the Office of Naval Research under Contract No. N0001488WX24207.

\section{References}

1. H. M. Smallwood, J. Appl. Phys. 15, 758 (1944).

2. E. Guth, Rubber Chem. Technol. 18, 596 (1945).

3. G. K. Batchelor and J. T. Green, J. Fluid Mech. 56, 401 (1972).

4. N. Nakajima, Rubber Chem. Technol. 61, 938 (1988).

5. A. Medalia, J. Colloid. Interface Sci. 32, 115 (1970).

6. G. Kraus, J. Polym. Sci. B 8, 601 (1970).

7. A. Medalia, Rubber Chem. Technol. 47, 411 (1974).

8. J. D. Ulmer, W. M. Hess, and V. E. Chirico, Rubber Chem. Technol. 47, 729 (1974)

9. E. M. Dannenberg, Trans. Inst. Rubber Ind. 42, 26 (1966).

10. F. Bueche, Physical Properties of Polymers (Wiley, New York, 1962).

11. J.-B. Donnet and A. Voet, Carbon Black-Physics, Chemistry and Elastomer Reinforcement (Dekker, New York, 1976).

12. A. Voet, A. K. Sircar, and T. J. Mullins, Rubber Chem. Technol. 42, 874 (1969).

13. A. Medalia, Rubber Chem. Technol. 60. 45 (1987).

14. Carbon Black-Polymer Composites-The Physics of Electrically Conducting Composites, edited by E. K. Sichel (Dekker, New York, 1982).

15. J. R. Nelson and W. K. Wissing, Carbon 24, 115 (1986).

16. A. I. Medialia, Rubber Chem. Technol. 59, 432 (1986)

17. A. I. Eatah, A. A. Ghani, and A. A. Hashem, Angew. Makromol. Chem. 165, 69 (1989).

18. J. L. White, in Mechanical Properties of Reinforced Thermoplastics, edited by D. W. Clegg and A. A. Collyer (Elsevier, London, 1987), Chap. 5.

19. K. Lakdawala and R. Salovey, Polym. Eng. Sci. 28, 877 (1988). 
20. A. R. Payne, J. Appl. Polym. Sci. 6, 57 (1962).

21. R. S. Waring, Trans. Inst. Rubber Ind. 26, 4 (1950)

22. K. E. Gui, C. S. Wilkinson, and S. D. Gehman, Ind. Eng. Chem. 44, 720 (1952).

23. A. R. Payne and R. F. Whittaker, Rubber Chem. Technol. 44, 440 (1971)

24. E. O. Forster, IEEE Trans. Power Appar. Syst. PAS-90, 913 (1971).

25. M. G. Siswanto, N. P. Bo, Parangtopo, and II. Neubacher, Rubber Chem. Technol. 61, 269 (1988)

26. A. R. Payne, J. Appl. Polym. Sci. 9, 1073 (1965).

27. A. Voet and F. R. Cook, Rubber Chem. Technol. 41, 1208 ( 1968 ).

28. D. R. Parris, L. C. Burton, and M. G. Siswanto, Rubber Chem. Technol. 60, 705 (1987).

29. A. I. Medalia, Rubber Chem. Technol. 51, 437 (1978)

30. A. R. Payne, J. Appl. Polym. Sci. 7, 873 (1963).

31. A. Voet and F. R. Cook, Rubber Chem. Technol. 40, 1364 (1967).

32. A. Voet and F. R. Cook, Rubber Chem. Technol. 41, 1215 (1968).

33. A. K. Sircar and T. G. Lamond, Rubber Chem. Technol. 48, 79 (1975).

34. A. K. Sircar and T. G. Lamond, Rubber Chem. Technol. 48, 89 (1975).

35. A. N. Gent and P. B. Lindley, Proc. Inst. Mech. Eng. (London) 173, 111 (1959).

36. S. S. Sternstein, Adv. Chem. Ser. 203, 123 (1983).

37. Y. Isono and J. D. Ferry, Rubber Chem. Technol. 57, 925 (1984).

38. W. P. Fletcher and A. N. Gent, Bri. J. Appl. Phys. 8, 194 (1957).

39. J. M. Funt, Rubber Chem. Technol. 61, 842 (1988).

40. P. Mason, J. Appl. Polym. Sci. 4, 212 (1960)

41. G. Kraus and J. T. Gruver, J. Polym. Sci. A2 8, 571 ( 1970).

42. C. M. Roland, J. Poly. Sci. Polym. Phys. Ed. 26, 839 (1988)

43. C. A. Trask and C. M. Roland, Macromolecules 22, 256 (1989).

44. C. M. Roland and C. A. Trask, Polym. Mater. Sci. Eng. 60, 832 (1989).

45. A. R. Payne, J. Appl. Polym. Sci. 8, 2661 (1964).

46. A. R. Payne, J. Appl. Polym. Sci. 16, 1191 (1972).

47. G. Kraus, J. Appl. Polym. Sci. Appl. Polym. Symp. 39, 75 (1984).

48. C. M. Roland, in Handbook of Elastomers - New Developments and Technology, edited by A. K. Bhowmick and H. L. Stephens (Dekker, New York, 1988), Chap. 6.

49. G. D. Dean, J. C. Duncan, and A. F. Johnson, Polym. Testing 4, 225 (1984).

50. M. Amin, H. H. Hassan, and E. M. Abdel-Bary, J. Polym. Sci. Polym. Chem. Ed. 12, 2691 (1974).

51. K. Arai and J. D. Ferry, Rubber Chem. Technol. 59, 592 (1986). 